\title{
Mentoring during anesthesia residency training: challenges and opportunities
}

\author{
Donald R. Miller, MD • Colin J. L. McCartney, PhD
}

Received: 28 May 2015/Accepted: 12 June 2015/Published online: 23 June 2015

(C) Canadian Anesthesiologists' Society 2015

\begin{abstract}
Mentorship: "The guidance provided by a mentor, especially an experienced person in a company or an institution." Oxford Dictionaries ${ }^{\mathrm{A}}$
\end{abstract}

Three key components are required for success at any stage of a medical career: an engaged physician, one or more experienced mentors, and the time to invest in learning. Mentorship is a vital component for success, and few would argue the benefits of having one or more experienced and engaged mentors during their career. In medicine, the many benefits of being mentored have been well established, and some such advantages include enhanced career planning, greater productivity and career satisfaction, and increased confidence as educators. ${ }^{1}$ Furthermore, there is a greater likelihood that those who are mentored will pursue an academic career and achieve higher academic deliverables in regard to promotion and scholarly activities, including peer-reviewed publication and grant procurement. ${ }^{2}$ Mentorship is also a key to the Royal College of Physicians and Surgeons of Canada CanMEDS Physician Competency Framework (http://www. royalcollege.ca/portal/page/portal/rc/common/documents/ canmeds/framework/framework_series_1_e.pdf) and should provide trainees with role models in such intrinsic roles as collaborator, communicator, and manager. Results of a recent survey of internal medicine residents in training

D. R. Miller, MD $(\bowtie) \cdot$ C. J. L. McCartney, PhD

Department of Anesthesiology, The Ottawa Hospital, The Ottawa Hospital Research Institute, General Campus, CCW 1409, 501 Smyth Road, Ottawa, ON K1H 8L6, Canada e-mail:dmiller@toh.on.ca

D. R. Miller, MD • C. J. L. McCartney, PhD

Faculty of Medicine, The University of Ottawa, Ottawa,

ON, Canada programs affiliated with Harvard Medical School showed that mentored residents were nearly twice as likely as their non-mentored counterparts to have excellent career preparation. ${ }^{3}$ At the same time, effective mentorship should be equally valuable to those choosing careers in community hospital settings by providing guidance in such areas as professionalism, personal growth and development, and work-life balance. Reciprocally, mentors can also benefit from mentorship programs by attaining leadership and coaching skills, the ability to reflect on personal performance, and the satisfaction of sharing experiences and knowledge.

Ideally, mentorship programs should be longitudinal; i.e., they should be integrated into medical school curricula and postgraduate training programs, but also extend to the junior faculty level. Despite the known and potential benefits, it is surprising that relatively little has been documented about mentorship specific to the specialty of anesthesia. ${ }^{4}$ At a time when our specialty has been challenged academically on several fronts, lack of data on mentorship during residency presents a notable problem. In an effort to close some of this knowledge gap, Zakus et al. present the results of two interesting surveys in this issue of the Journal. ${ }^{5}$ These authors developed two related surveys to determine the prevalence of formal mentorship programs in anesthesiology training programs in Canada and to ascertain the overall prevalence of mentorship at the level of the postgraduate trainee. For the purpose of their study, a formal mentorship program was defined as "one in which there is oversight by the program director or designated faculty and the program facilitates and

\footnotetext{
$\overline{\mathrm{A}}$ http://www.oxforddictionaries.com/definition/learner/mentor (accessed June 2015).
} 
encourages matching of mentees and mentors". Anesthesia training programs in Canada are five years in duration, and presumably, mentoring programs, where they exist, would span the entire five-year training period. Using two validated survey instruments (one for program directors and one for residents), the authors sought to determine what key factors would guide whether a resident would have an identified mentor during their five-year training. It was shown that, as of 2014, formal anesthesia mentorship programs in anesthesia were being administered in slightly more than half $(54 \%)$ of the 16 anesthesia residency training programs in Canada. While over $94 \%$ of resident respondents identified that mentorship during residency training is important, it is disappointing that $42 \%$ of respondents indicated that they did not interact regularly (i.e., either face-to-face or via e-mail, telephone, or internet video) with their mentor. Unsurprisingly, the authors found that residents were much more likely to have a mentor during residency when a formal mentorship program was in place. The most frequent areas of mentor-mentee discussion included career planning and goals, clinical expert roles, work-life balance, and exams. Other important themes that were somewhat less frequently discussed were research, professionalism and teaching, and finally, personal finance matters.

The authors address several limitations of the survey, most notably the overall resident response rate that was only 39\% (203/519). ${ }^{5}$ The relatively low response rate (although not unusual for an e-mail survey) makes the sampling frame subject to potential bias, and the prevalence of mentorship may have been overestimated. In addition, although distributed to all English and French training programs in Canada, the survey instrument was written exclusively in English. Furthermore, it is difficult to obtain an accurate description of the mentorship characteristics of the mentors, including their training and experience in mentoring. Finally, the impact of the mentorship program on professional, personal, and academic outcomes during and following residency cannot be assessed without longitudinal follow-up. Despite the above-noted limitations, the authors are to be congratulated for undertaking an important study that should be equally relevant to anesthesia residents, programs directors, clinical and research faculty, and department chairs in anesthesia across Canada and internationally.

The Merriam-Webster Dictionary provides a rather broad definition of mentor as "someone who teaches or gives help and advice to a less experienced and often younger person." $\mathrm{B}$

\footnotetext{
B www.merriam-webster.com/dictionary/mentor (accessed June 2015).
}

Mentors may serve to some extent as advisors, role models, and teachers. Key elements include the importance of an ability to share confidential feedback that is not used in assessment and a solid trust between mentor and mentee. The importance of having skilled mentors in a mentorship program cannot be overestimated. Whereas some of these skills may be acquired naturally, other mentoring skills may be further developed through formal training or coaching. Cho et al. recently examined the important qualities of mentors as described by their mentees' letters of nomination for a lifetime achievement award in mentorship. They identified five key attributes of an attending physician that would characterize an excellent role model: "(1) admirable personal qualities, including enthusiasm, compassion, and selflessness; (2) act as a career guide, offering a vision but purposefully tailoring support to each mentee; (3) make strong time commitments with regular, frequent, and high-quality meetings; (4) support personal/professional balance; and (5) leave a legacy of being a good mentor through role modeling and instituting policies that set global expectations and standards for mentorship." 6 Whereas these findings applied to mentoring junior faculty in an academic health sciences centre, the underlying tenets should be broadly applicable to mentoring programs for residents as well. With regard to role modeling, nearly two decades ago, a case-control study of internal medicine house staff at four North American teaching hospitals showed that the following attributes were independently associated with being an excellent physician role model: spending more than $25 \%$ of one's time in teaching, stressing the importance of the doctor-patient relationship in one's teaching, teaching the psychosocial aspects of medicine, and having served as a chief resident. ${ }^{7}$ Importantly, most of the attributes associated with being an excellent role model are skills that can be learned.

Program directors play a key role in promoting and ensuring the success of resident mentorship programs. It needs to be recognized, however, that those involved in assessment will often have difficulty providing a true mentor-mentee relationship because of the inevitable conflict between the roles of assessor and mentor. The nature of the support, orientation, and training, if any, that program directors receive to run their mentorship programs is unclear. While recognizing the existence of seven competencies in the recently updated CanMEDS 2015 Physician Competency Framework, traditional residency curricula continue to place considerable emphasis on training and evaluation of medical expert competencies. Within the limits of educational resources (time, money, faculty resources), formal mentoring of residents in the six so-called "intrinsic roles" (e.g., communicator, collaborator, professional) may not always be considered 
a priority. It is interesting that $24 \%$ of program directors did not respond to the survey of Zakus et al., and nearly half $(46 \%)$ of anesthesia residency training programs in Canada lack a formal mentoring program. ${ }^{5}$ Parallel observations in internal medicine have been reported by Castiglioni et al. In their survey of internal medicine program directors published in 2004, these authors found that, whereas over $60 \%$ of program directors favoured mentoring, only $49 \%$ of internal medicine residencies have mentoring programs, and overall, mentoring programs are largely unstructured, loosely monitored, and underevaluated. $^{8}$

High-quality hypothesis-driven research programs that address important clinical questions are important to the growth of all medical specialties. The traditional five-year postgraduate training period (soon to be four years at the University of Ottawa) presents a unique opportunity for residents possibly considering a clinician-researcher or clinician-investigator career path to be mentored in fundamental aspects of research. In 2006, a survey report by Silcox et al. provided a Canadian perspective on the attitudes of residents and program directors towards research during anesthesiology training. In their report, these authors identified a number of barriers to residents undertaking a research project. ${ }^{9}$ One key element was inadequate faculty support and mentorship. We recognize and appreciate that a small percentage of anesthesia residents will proceed along a career path that integrates either clinical or basic science research. Nevertheless, integrating opportunities for residents to participate in research during residency training, mentoring in academic integrity, ${ }^{10}$ and encouraging interested residents to present at major conferences and to publish their research requires a core of engaged and knowledgeable research mentors in every academic department of anesthesia. ${ }^{11}$

Finally, formal mentoring should be a continuum through one's career and should not conclude at the end of residency. At the University of Ottawa, Department of Anesthesia, we have recently established an early career faculty mentorship program to support the success and development of new faculty members to develop professionally as outstanding academic anesthesiologists. This program aims to provide a supportive, collaborative, and collegial environment to take new recruits and support them to success in their early academic career. Inherent in the mission is the explicit recognition that individual success will require department-wide support and that such success will be shared by the mentees, their mentors, and the Department. The spirit of the program is to encourage and support academic development of new faculty through the provision of structure, resources, advice, written expectations, and defined achievable endpoints. The program is three years in duration for each mentee and applies to all newly appointed lecturers and assistant professors at the University of Ottawa, Department of Anesthesia. After three years and after meeting the predefined goals, annual reviews with the head of anesthesia or a delegate shall continue as for all faculty members of the Department. There will be expectations that intermediate-career and senior faculty will share their experience and expertise and be engaged in the growth of the new generation of faculty. A culture of shared values regarding contributions to academic productivity at the University of Ottawa will be promoted. Experiences will be shared through the Association of Canadian University Departments of Anesthesia, and in due course, through peer-reviewed publication.

In conclusion, while there is a body of evidence showing the importance of mentorship during residency training, Zakus et al. provide important data that quantify several important challenges which currently limit universal adoption of robust mentorship programs in the specialty of anesthesia. The opportunities are considerable; moreover, the new direction towards competency-based education in Canada and elsewhere has bolstered the rationale to commit resources and training within each academic department in order to develop, enhance, and monitor mentoring programs for both residents and faculty. Chairs of anesthesia and residency program directors should be encouraged to collaborate on mutual goals and objectives of mentorship programs for anesthesia residents. The benefits of providing structured support for residents and junior faculty should promote our academic mission and, in due course, enhance care for our patients.

\section{Le mentorat pendant la résidence en anesthésie: défi et opportunités}

\author{
Mentorat : "L'orientation offerte par un mentor, \\ particulièrement une personne d'expérience dans une \\ société ou une institution. " Traduction de la \\ définition du Oxford Dictionary ${ }^{\mathrm{A}}$
}

Trois composantes clés sont indispensables à la réussite, quel que soit le niveau où l'on se trouve dans notre carrière médicale : être impliqué dans sa pratique, avoir un ou plusieurs mentor(s) d'expérience et du temps à investir dans l'apprentissage. Le mentorat est essentiel à la réussite, et rares sont ceux qui nieront les avantages d'avoir pu bénéficier du soutien d'un ou plusieurs mentors impliqués et expérimentés au cours de leur carrière. En médecine, les 
nombreux avantages du mentorat sont bien établis. Citons par exemple une meilleure planification de carrière, une plus grande productivité et une meilleure satisfaction professionnelle, ainsi qu'une confiance accrue en notre qualité de formateurs. ${ }^{1}$ En outre, les personnes ayant eu un mentor sont plus enclines à poursuivre une carrière universitaire et à atteindre des objectifs académiques plus élevés en matière de promotion et d'activités universitaires, y compris la publication dans des revues révisées par des pairs et l'obtention de bourses. ${ }^{2}$ Le mentorat constitue également une clé du Cadre de compétences CanMEDS pour les médecins du Collège royal des médecins et chirurgiens du Canada (http://www.royalcollege.ca/portal/ page/portal/rc/common/documents/canmeds/framework/ framework_series_1_f.pdf) et devrait offrir aux résidents des modèles à émuler dans des rôles intrinsèques tels que ceux de collaborateur, de communicateur ou encore de gestionnaire. Les résultats d'un sondage récent auprès de résidents en médecine interne dans des programmes de formation affiliés à l'École de médecine de Harvard ont montré que les résidents ayant un mentor avaient deux fois plus de chances que leurs pendants sans mentor de bénéficier d'une excellente préparation professionnelle. ${ }^{3}$ Ceci étant dit, un mentorat efficace devrait être tout aussi utile aux personnes choisissant de poursuivre une carrière dans un hôpital communautaire en offrant des conseils dans des domaines tels que le professionnalisme, le développement et la croissance personnels, et l'équilibre travail - vie privée. La réciproque est également vraie : les mentors peuvent eux aussi tirer profit des programmes de mentorat en acquérant des compétences de leadership et de formation, en ayant la possibilité de réfléchir à leurs performances personnelles et en ressentant la satisfaction de partager leurs expériences et leurs connaissances.

Dans l'idéal, les programmes de mentorat devraient être longitudinaux, c'est-à-dire qu'ils devraient être intégrés dans les programmes de l'école de médecine et les programmes de formation postdoctorale, mais également s'étendre jusqu'aux premières années de carrière. Malgré les avantages connus et potentiels du mentorat, il est surprenant de constater que ce type d'activités est relativement peu documenté dans la spécialité de l'anesthésie. ${ }^{4}$ Alors que notre spécialité est confrontée à plusieurs défis d'un point de vue académique, l'absence de données sur le mentorat pendant la résidence constitue un problème de taille. Dans un effort pour combler ces lacunes dans nos connaissances, Zakus et coll. présentent, dans ce numéro du Journal, les résultats de deux sondages intéressants. Ces auteurs ont mis au point deux questionnaires liés afin de déterminer la prévalence des programmes formels de mentorat dans les programmes de formation en anesthésiologie au Canada et d'examiner la prévalence globale du mentorat au niveau des étudiants postgradués. Aux fins de leur étude, un programme formel de mentorat était défini comme suit : « un [programme] dans lequel il y a supervision par le directeur du programme ou un membre désigné du corps professoral et qui facilite et encourage la création de paires mentoré-mentor. » Les programmes de résidence en anesthésie durent cinq ans au Canada; on prend donc pour acquis que les programmes de mentorat, là où ils existent, durent la totalité de la période de formation de cinq ans. À l'aide de deux instruments de sondage validés (l'un destiné aux directeurs de programme et l'autre aux résidents), les auteurs ont cherché à identifier les facteurs clés qui détermineraient la présence d'un mentor dans la vie du résident pendant ces cinq années de formation. Les auteurs ont démontré que, en date de 2014, il existait des programmes formels de mentorat en anesthésie dans un peu plus de la moitié (54\%) des programmes de résidence en anesthésie au Canada. Alors que le mentorat pendant la résidence était considéré comme important par $94 \%$ des résidents ayant répondu au sondage, il est décevant que 42 $\%$ des répondants aient indiqué qu'ils n'interagissaient pas de façon régulière (soit face à face, par courriel, téléphone ou vidéoconférence) avec leur mentor. Sans surprise, les auteurs ont observé qu'il était beaucoup plus probable que les résidents aient un mentor pendant leur résidence si un programme formel de mentorat existait dans leur institution. Les questions les plus souvent abordées par les mentorés avec leurs mentors étaient la planification et les objectifs de carrière, les rôles d'expert clinique, l'équilibre vie privée - vie professionnelle, et les examens. Parmi les autres sujets importants mais moins souvent abordés figuraient la recherche, le professionnalisme, l'enseignement et, finalement, les questions de finances personnelles.

Les auteurs abordent plusieurs des écueils de leur sondage, le plus frappant étant le taux global de réponse des résidents, de $39 \%$ seulement (203/519). ${ }^{5}$ Ce taux de réponse relativement faible (bien qu'assez fréquent lors de sondage par courriel) rend le cadre d'échantillonnage susceptible à un biais potentiel, et la prévalence du mentorat pourrait donc avoir été surestimée. De plus, bien qu'il ait été distribué dans tous les programmes de résidence anglophones et francophones au Canada, le sondage n'était rédigé qu'en anglais. En outre, il est difficile d'obtenir une description précise des caractéristiques de mentorat des mentors, y compris leur formation et leur expérience de mentorat. Enfin, l'impact du programme de mentorat sur les résultats professionnels, personnels et universitaires pendant et après la résidence ne peut être évalué sans suivi longitudinal. Malgré ces écueils, il convient de féliciter les auteurs d'avoir entrepris une étude importante, pertinente tant pour les résidents en anesthésie, les directeurs de programme, les patrons de 
clinique et de recherche, que les chaires des départements d'anesthésie au Canada et ailleurs dans le monde.

Le dictionnaire anglais Merriam-Webster propose une définition assez floue du mentor: " une personne qui enseigne ou offre de l'aide et des conseils à une personne moins expérimentée et souvent plus jeune. ${ }^{\mathrm{B}}$

Les mentors peuvent, dans une certaine mesure, servir de conseillers, de rôles à émuler, ou encore d'enseignants. Parmi les éléments clés d'un bon mentorat figurent le fait de pouvoir partager des rétroactions confidentielles qui ne sont pas utilisées dans l'évaluation et ainsi qu'une solide confiance entre le mentoré et le mentor. Il est absolument essentiel de disposer de mentors compétents dans un programme de mentorat. Bien que certaines compétences puissent être innées, d'autres compétences de mentorat peuvent être acquises et développées grâce à une formation ou un coaching formel. Cho et coll. ont récemment examiné les qualités cruciales que devrait posséder un mentor en basant leur définition sur les lettres de nomination de mentorés pour un Prix de mérite en mentorat. Ces auteurs ont identifié cinq attributs clés d'un médecin en pratique qui caractériseraient un excellent modèle à émuler : «(1) des qualités personnelles admirables, notamment l'enthousiasme, la compassion et l'altruisme; (2) le fait d'agir comme guide de carrière, en offrant une vision mais en personnalisant le soutien à chaque mentoré; (3) le fait de mettre beaucoup de temps à disposition pour des réunions régulières, fréquentes et de qualité; (4) l'encouragement d'un équilibre vie privée - vie professionnelle; et (5) un héritage de bon mentor en montrant par l'exemple et en mettant en œuvre des politiques afin de déterminer des attentes et des normes globales de mentorat. ${ }^{6}$ Bien que ces données se fondent sur le mentorat de jeunes médecins dans un centre universitaire de sciences de la santé, les principes sous-jacents devraient aussi être applicables - dans les grandes lignes - aux programmes de mentorat de résidents. En ce qui concerne les modèles à émuler, il y a près de 20 ans, une étude de cas-témoin de personnel de médecine interne a été réalisée dans quatre hôpitaux universitaires nord-américains. L'étude a déterminé que les attributs suivants étaient associés de façon indépendante au fait d'être considéré comme un excellent modèle de médecin: le fait de passer plus de $25 \%$ de son temps à enseigner, de souligner l'importance de la relation médecin-patient dans son enseignement, d'enseigner les aspects psychosociaux de la médecine, et d'avoir été résident en chef. ${ }^{7}$ Notons que la plupart de ces attributs peuvent être appris.

Les directeurs de programme jouent un rôle clé pour promouvoir et garantir la réussite des programmes de mentorat destinés aux résidents. Cependant, il est important de souligner que les personnes impliquées dans l'évaluation des résidents auront souvent de la difficulté à offrir une véritable relation de mentor à mentoré en raison du conflit inévitable entre le rôle d'évaluateur et celui de mentor. La nature du soutien, de l'orientation et de la formation, le cas échéant, que reçoivent les directeurs de programme pour gérer les programmes de mentorat est peu claire. Tout en reconnaissant l'existence de sept compétences dans le Cadre de compétence CanMEDS 2015 pour les médecins récemment mis à jour, les programmes conventionnels de résidence continuent à mettre l'accent sur la formation et l'évaluation des compétences médicales expertes. Tout en respectant les limites des ressources éducationnelles (le temps, l'argent et les ressources en personnel), le mentorat formel des résidents dans les six rôles dits 'intrinsèques' (par ex., communicateur, collaborateur, professionnel) pourrait ne pas toujours être considéré comme une priorité. Il est intéressant de noter que $24 \%$ des directeurs de programme n'ont pas répondu au sondage de Zakus et coll., et près de la moitié $(46 \%)$ des programmes de résidence en anesthésie au Canada ne disposent pas d'un programme formel de mentorat. ${ }^{5}$ Castiglioni et coll. ont rapporté des taux semblables en médecine interne. Dans leur sondage des directeurs de programme de médecine interne publié en 2004, ces auteurs ont découvert que, bien que $60 \%$ des directeurs de programme aient été en faveur du mentorat, seuls $49 \%$ des programmes de résidence en médecine interne disposaient de programmes de mentorat et que, globalement, les programmes de mentorat manquaient de structure, de supervision et d'évaluation. ${ }^{8}$

Des programmes de recherche fondée sur des hypothèses de haute qualité et qui traitent de questions cliniques fondamentales sont essentiels à la croissance de toutes les spécialités médicales. La période traditionnelle de formation postdoctorale de cinq ans (bientôt de quatre ans à l'Université d'Ottawa) constitue une occasion unique pour les résidents envisageant peut-être une carrière de clinicien-chercheur ou de clinicien-investigateur d'être guidés par un mentor en ce qui touche aux aspects fondamentaux de la recherche. En 2006, un sondage de Silcox et coll. proposait une perspective canadienne sur les attitudes des résidents et des directeurs de programme envers la recherche pendant la formation en anesthésiologie. Dans leur article, ces auteurs ont identifié plusieurs obstacles auxquels étaient confrontés les résidents s'attelant à un projet de recherche. ${ }^{9}$ L'un des problèmes majeurs était le manque de soutien des professeurs et un manque de mentorat. Nous sommes conscients et tenons compte du fait qu'un faible pourcentage de résidents en anesthésie seulement poursuivra une carrière intégrant de la recherche en clinique ou en science fondamentale. Toutefois, si l'on souhaite créer des occasions pour les résidents de participer à des projets de recherche pendant leur résidence, les 
guider en matière d'intégrité universitaire, ${ }^{10}$ et encourager ceux qui sont intéressés à présenter des articles lors de congrès d'envergure et à publier leurs recherches, il faut disposer d'un noyau de mentors de recherche impliqués et bien informés dans chaque département universitaire d'anesthésie. ${ }^{11}$

Enfin, le mentorat formel devrait perdurer tout au long de la carrière et non pas s'interrompre à la fin de la résidence. $\mathrm{Au}$ département d'anesthésie de l'Université d'Ottawa, nous avons récemment mis sur pied un programme de mentorat pour les anesthésiologistes en début de carrière afin de soutenir la réussite et le développement des nouveaux membres du département pour qu'ils s'épanouissent sur le plan professionnel et deviennent des anesthésiologistes universitaires d'exception. Ce programme a pour but d'offrir un environnement de soutien, de collaboration et de collégialité afin d'intégrer les nouvelles recrues et de les encourager à réussir le début de leur carrière universitaire. Ce faisant, nous reconnaissons de manière explicite que la réussite individuelle nécessite un soutien de la part du département dans son intégralité; de plus, une telle réussite sera partagée par les mentorés, leurs mentors et le département au complet. L'idée du programme est d'encourager et de soutenir le développement académique des nouveaux membres du département en leur fournissant la structure, les ressources, les conseils, des attentes écrites et des objectifs atteignables prédéfinis. Le programme dure trois ans pour chaque mentoré et s'applique à tous les nouveaux chargés de cours et professeurs adjoints du département d'anesthésie de l'Université d'Ottawa. Après trois ans et après avoir atteint leurs objectifs prédéfinis, des bilans annuels avec le chef du département ou un délégué se poursuivront, comme c'est le cas pour tous les membres du département. On attend des membres plus anciens qu'ils partagent leurs expériences et leur expertise et qu'ils s'impliquent dans la formation de la nouvelle génération d'anesthésiologistes. Une culture de valeurs partagées quant aux contributions à la productivité universitaire à l'Université d'Ottawa sera encouragée. Nos expériences seront partagées via l'Association des Départements d'anesthésie des universités canadiennes et, en temps voulu, via une publication dans une revue révisée par les pairs.

Pour conclure, bien qu'il existe de nombreuses données probantes soutenant l'importance du mentorat pendant la résidence, Zakus et coll. proposent des données importantes qui permettent de quantifier plusieurs défis importants qui limitent actuellement l'adoption universelle de programmes rigoureux de mentorat dans la spécialité de l'anesthésie. Les occasions ne manquent pas; de plus, la nouvelle orientation vers une formation basée sur les compétences au Canada et ailleurs encourage et justifie l'allocation de ressources et de formation dans chaque département universitaire afin de mettre au point, d'améliorer et de superviser des programmes de mentorat pour les résidents aussi bien que les patrons. Les chaires d'anesthésie et les directeurs de programme de résidence doivent être encouragés à collaborer pour atteindre les buts et objectifs mutuels des programmes de mentorat pour les résidents en anesthésie. Les avantages de procurer un soutien structuré aux résidents et aux nouveaux membres du département devraient promouvoir notre mission universitaire et, en temps voulu, améliorer les soins prodigués à nos patients.

Competing interests None declared.

Conflits d'intérêt aucun.

\section{References}

1. Sambunjak D, Straus SE, Marusic A. Mentoring in academic medicine: a systematic review. JAMA 2006; 296: 1103-15.

2. Berk RA, Berg J, Mortimer R, Walton-Ross B, Yeo TP. Measuring the effectiveness of faculty mentoring relationships. Acad Med 2005; 80: 66-71.

3. Ramanan RA, Taylor WC, Davis RB, Philipps RS. Mentoring matters. Mentoring and career preparation in internal medicine residency training. J Gen Intern Med 2006; 21: 340-5.

4. Flexman AM, Gelb AW. Mentorship in anesthesia: how little we know. Can J Anesth 2012; 59: 241-5.

5. Zakus $P$, Gelb AW, Flexman AM. A survey of mentorship among Canadian anesthesiology residents. Can J Anesth 2015; 62: this issue. DOI:10.1007/s12630-015-0418-8.

6. Cho CS, Ramanan RA, Feldman MD. Defining ideal qualities of mentorship: a qualitative analysis of the characteristics of outstanding mentors. Am J Med 2011; 124: 453-8.

7. Wright SM, Kern DE, Kolodner K, Howard DM, Brancati FL. Attributes of excellent attending-physician role models. N Engl J Med 1998; 339: 1986-93.

8. Castiglioni A, Bellini LM, Shea JA. Program directors' views of the importance and prevalence of mentoring in internal medicine residencies. J Gen Intern Med 2004; 19: 779-82.

9. Silcox LC, Ashbury TL, VanDenKerkhof EG, Milne B. Residents' and program directors' attitudes toward research during anesthesiology training: a Canadian perspective. Anesth Analg 2006; 102: 859-64.

10. Ochroch EA, Eckenhoff $R G$. The role of mentoring in aiding academic integrity. Anesth Analg 2011; 112: 732-4.

11. Beattie WS, Donati F, Cheng D, Mazer D, Miller DR. From concept to publication: celebrating mentorship and knowledge generation though research during postgraduate residency training. Can J Anesth 2013; 60: 625-9. 